\title{
A first case report of nasopharyngeal Mycobacterium abscessus subspecies massiliense infection
}

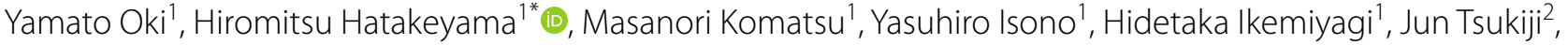 \\ Ryoko Higa ${ }^{2}$ and Nobuhiko Oridate ${ }^{3}$
}

\begin{abstract}
Background: Mycobacterium abscessus subspecies massiliense is a non-tuberculous mycobacteriosis and was subdivided from Mycobacterium abscessus in 2006. This article is the first report on nasopharyngitis caused by Mycobacterium abscessus subspecies massiliense.

Case presentation: A 45-year-old woman had an 18-month history of recurrent nasopharyngitis and presented with pain in the throat. Mycobacterial tissue culture and polymerase chain reaction testing revealed the presence of Mycobacterium abscessus subspecies massiliense in the nasopharyngeal tissue. This patient underwent surgery, followed by multiple rounds of chemotherapy with oral and intravenous antibiotic agents for 16 weeks. She has had no recurrence during the 56 weeks since treatment.

Conclusion: It is difficult to detect the presence of Mycobacterium abscessus subspecies massiliense in a culture from the swabbing sample. The tissue culture from a biopsy specimen is mandatory for the identification of the species. Currently, no definite treatment policy is available and only empirical treatment is applied. This case is an important for the diagnosis and treatment of this bacterial infection on nasopharynx.
\end{abstract}

Keywords: Mycobacterium massilliense, Mycobacterium abscessus subspecies massiliense, Nasopharyngitis, Nontuberculosis mycobacterium, NTM, Mycobacterium abscessus complex, Case report

\section{Background}

Mycobacterium abscessus subspecies massiliense (referred to hereafter as $M$. massiliense) is a non-tuberculous mycobacterosis (NTM) and is included among the rapidly growing mycobacteria (RGM) [1]. It was subdivided from Mycobacterium abscessus (M. abscessus) in 2006 [2].

M. massiliense is rare species described in only a few reports $[3,4]$. The $M$. abscessus complex, including $M$. massiliense, commonly causes skin, soft tissue as well as pulmonary infections [5]. Although there are reports

\footnotetext{
*Correspondence: htkym@yokohama-cu.ac.jp

1 Department of Otolaryngology, Yokohama City University Medical Center, Urafune-cho 4-57, Minami-ku, Yokohama 232-0024, Japan

Full list of author information is available at the end of the article
}

of $M$. massiliense infection in the head and neck region, such as otitis media [6], there are no reports of nasopharyngitis caused by this pathogen. We here report, to our knowledge, the first case of nasopharyngeal M. massiliense infection.

\section{Case presentation}

A 45-year-old woman was referred to Yokohama City University Medical Center with an 18-month history of recurrent nasopharyngitis. She presented with pain in the throat. Nasopharyngitis had been identified on a previous visit to an otolaryngology clinic. It had been temporarily improved by antibacterial treatment and nasopharyngeal abrasive therapy, which involves rubbing the nasopharynx with a nasal swab or pharyngeal cotton original author(s) and the source, provide a link to the Creative Commons licence, and indicate if changes were made. The images or other third party material in this article are included in the article's Creative Commons licence, unless indicated otherwise in a credit line to the material. If material is not included in the article's Creative Commons licence and your intended use is not permitted by statutory regulation or exceeds the permitted use, you will need to obtain permission directly from the copyright holder. To view a copy of this licence, visit http://creativecommons.org/licenses/by/4.0/. The Creative Commons Public Domain Dedication waiver (http://creativeco mmons.org/publicdomain/zero/1.0/) applies to the data made available in this article, unless otherwise stated in a credit line to the data. 
thread soaked in zinc chloride solution or compound iodine glycerin $[7,8]$. However, it recurred within a few weeks, and nasopharyngeal abrasive therapy continued for 12 weeks before the first visit. She was then referred to our hospital for further examination and treatment.

At presentation, she had infectious swelling of the adenoid remnant and pus on the lesion (Fig. 1a). Her physical examination was otherwise unremarkable and she had no other medical history including immunodeficiency.

The first pharyngeal swab culture test, which was gotten from epipharynx to insert the swab through the anterior nasal cavity, showed normal flora at the time of first visit. The same day, histopathological examination of a nasopharyngeal biopsy sample revealed the possibility of mycobacterial infection based on positive results for acidfast bacillus and Ziehl-Neelsen staining. Three weeks later, the first examination by pharyngeal mycobacteria culture from the sample which gotten by swabbing was

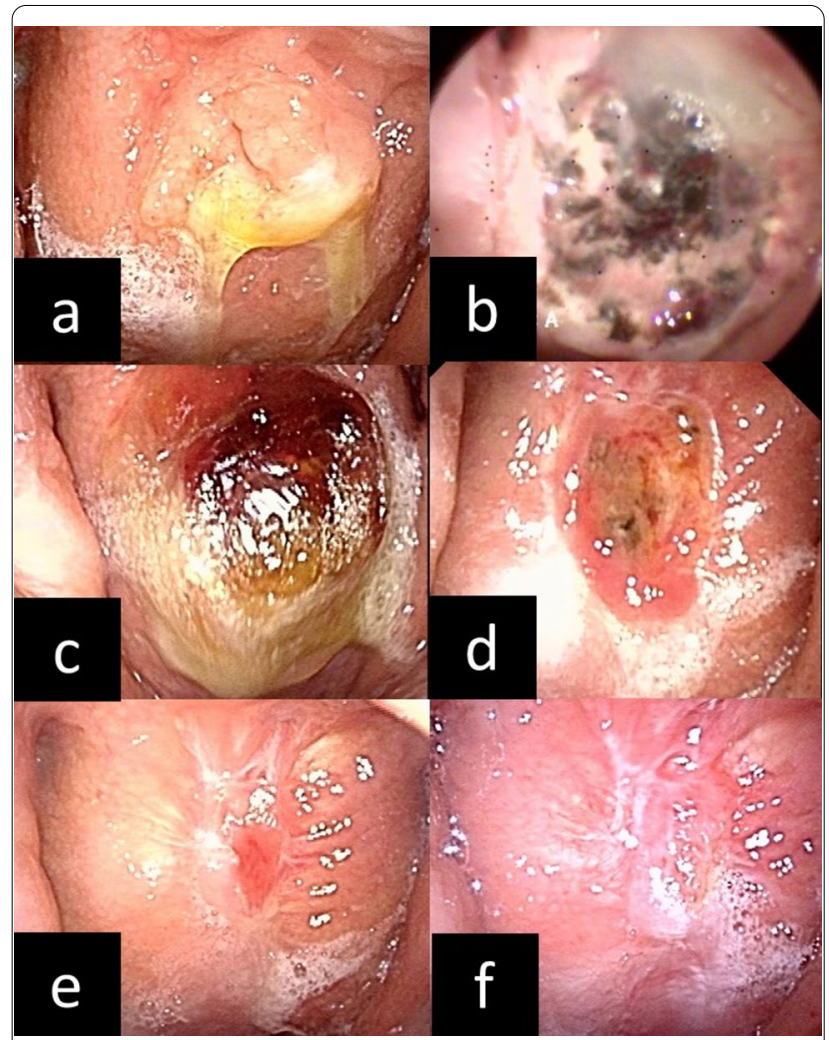

Fig. 1 a Findings of the nasopharynx before treatment. There were a raised nasopharyngeal lesion and a pus on the lesion. $\mathbf{b}$ Findings of the nasopharynx after adenoidectomy. Fur and black scabs for hemostasis had been attached to the wound. $\mathbf{c} 2$ weeks later after surgery. There was a pus on the lesion. The pus on wound was appropriately removed by dealing and washed. $\mathbf{d} 4$ weeks later after surgery. There was granulation rising in the wound. e 12 weeks later after surgery. The granulation remained but the wound was smaller gradually. $\mathbf{f} 20$ weeks later after surgery. The wound is epithelialized and scarred positive for smear dyeing of acid-fast bacillus stain, while the culture was negative. Four weeks later from first visit, we got the second mycobacterial culture from the biopsy specimen collected from nasopharynx using the sterile nasal cupped forceps with the visual guide of endoscope. It was wrapped in sterile gauze moistened with raw food to avoid exposure to tap water and transported promptly to the laboratory. It revealed the presence of $M$. abscessus complex using the MGIT ${ }^{\mathrm{TM}}$ System. (Becton and Dickinson Co. Mountain View, CA) All strains were classified as $M$. abscessus complex using a DDH Mycobacteria kit (Kyokuto Pharmaceutical Industrial, Tokyo, Japan) or by MALDI-TOF MS (Bruker Daltonics, Billerica, MA, USA). Mycobacterium abscessus subspecies abscessus, M. massiliense and Mycobacterium abscessus subspecies bolletii type strains were obtained from the Japan Collection of Microorganisms of the Riken Bio-Resource Center (BRC-JCM; Ibaraki, Japan). All bacterial strains/ isolates were subcultured on $2 \%$ Ogawa egg slants or 7H10 agar plates supplemented with 10\% OADC. All procedures were performed in a laminar flow cabinet to avoid contamination. PCR assays were done for differentiating Mycobacterium abscessus subspecies abscessus, M. massiliense, and Mycobacterium abscessus subspecies bolletii, as described previously [9], 10, and identified this species as M. massiliense (Fig. 2). The susceptibility test was not performed this case. This is because there is a difference between the in vitro results and the behaviors under antimicrobial use, and the information is for limited reference only $[1,10]$.

The patient underwent surgery to remove the inflamed adenoid tissue in the nasopharynx and washed the

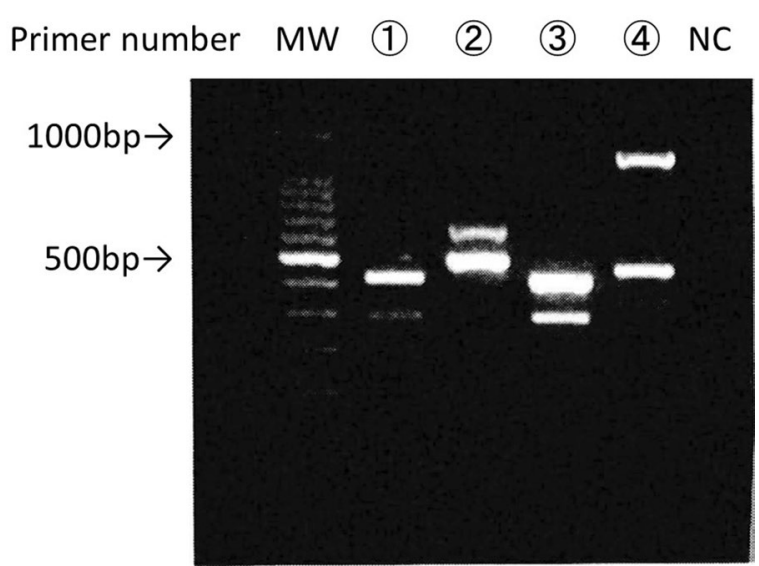

Fig. 2 Examination showed the disease as Mycobacterium abscessus subspecies massiliense infection. MW: molecular weight, (1) Sample of this case, (2) Mycobacterium abscessus subspecies abscessus, (3) Mycobacterium abscessus subspecies massiliense, (4) Mycobacterium abscessus subspecies bolletii. NC negative control 
wound with saline. At the same time, she was treated with oral clarithromycin at $400 \mathrm{mg} /$ day, intravenous imipenem at $2 \mathrm{~g} /$ day divided into four doses, and intravenous amikacin at $15 \mathrm{mg} / \mathrm{kg} /$ day three times a week. She received multiple antibacterial therapy for 2 weeks in an inpatient setting. She was then treated with oral clarithromycin at the same dosage, and intravenous amikacin two times a week after discharge. The treatment courses are summarized in Fig. 3. She received this combination therapy with clarithromycin and amikacin for an additional 4 months. The mycobacterial "tissue" culture was negative 8 weeks after surgery. She is currently being followed up in our hospital, and has had no adverse event and no recurrence during the 56 weeks since the cessation of treatment.

\section{Conclusions and discussion}

\section{Disease from M. massiliense and its infectious route}

Due to the low incidence of mycobacterial infections themselves, statistically, while there is a lot of information on the pulmonary region of $M$. massiliense, which accounts for about $0.6 \%$ of all NTM infections, $M$. massiliense infections in the head and neck region have rarely been reported [4]. As far as we are aware, this patient is the first case of pharyngeal $M$. massiliense infection.

It has been reported that infections caused by the $M$. abscessus complex usually follow accidental trauma or surgery [11]. On the other hand, multiple iatrogenic cases of NTM infection have been described in the past 2 decades [1], including cases resulting from cardiac surgery, injections, plastic surgery, middle ear tympanostomy tube replacement, and a variety of miscellaneous surgical procedures [1, 12-18]. Most of the health care-associated mycobacterial outbreaks have involved RGM, especially the M. abscessus complex and Mycobacterium fortuitum [19]. The common factor in health care-associated outbreaks is presumed to be exposure of a susceptible individual to an NTM-infected liquid, usually tap water [1]. As it may have been overlooked due to a low detection rate in culture tests, although NTM infection is rare its possible presence must still be considered.

The $M$. abscessus complex is also frequently detected in water and soil, and, in general, there are many cases of skin and soft tissue infections associated with trauma or surgery $[11,20]$.

NTMs are widely distributed in the environment [21, 22], and it is considered that NTM infection in humans is caused by bacterium from the environment [4]. As NTMs originally have only weak pathogenicity in humans, it parasitizes and infects humans. Therefore, infections are caused by some trigger, such as immunodeficiency [4]. On the other hand, some patients without obvious immunodeficiency develop NTM disease [23].

This case was a 45-year-old, previously healthy woman who was not immunodeficient. She should, therefore, be less likely to get infected. However, she had undergone nasopharyngeal abrasive therapy for 12 weeks prior to visit to our hospital. The purpose of this therapy is sterilization and disinfection using a coating agent and phlebotomy $[7,8]$. It is said that the greater the degree of disinfection, the higher the amount of bleeding, but the more effective it is. It is possible that the patient, who was

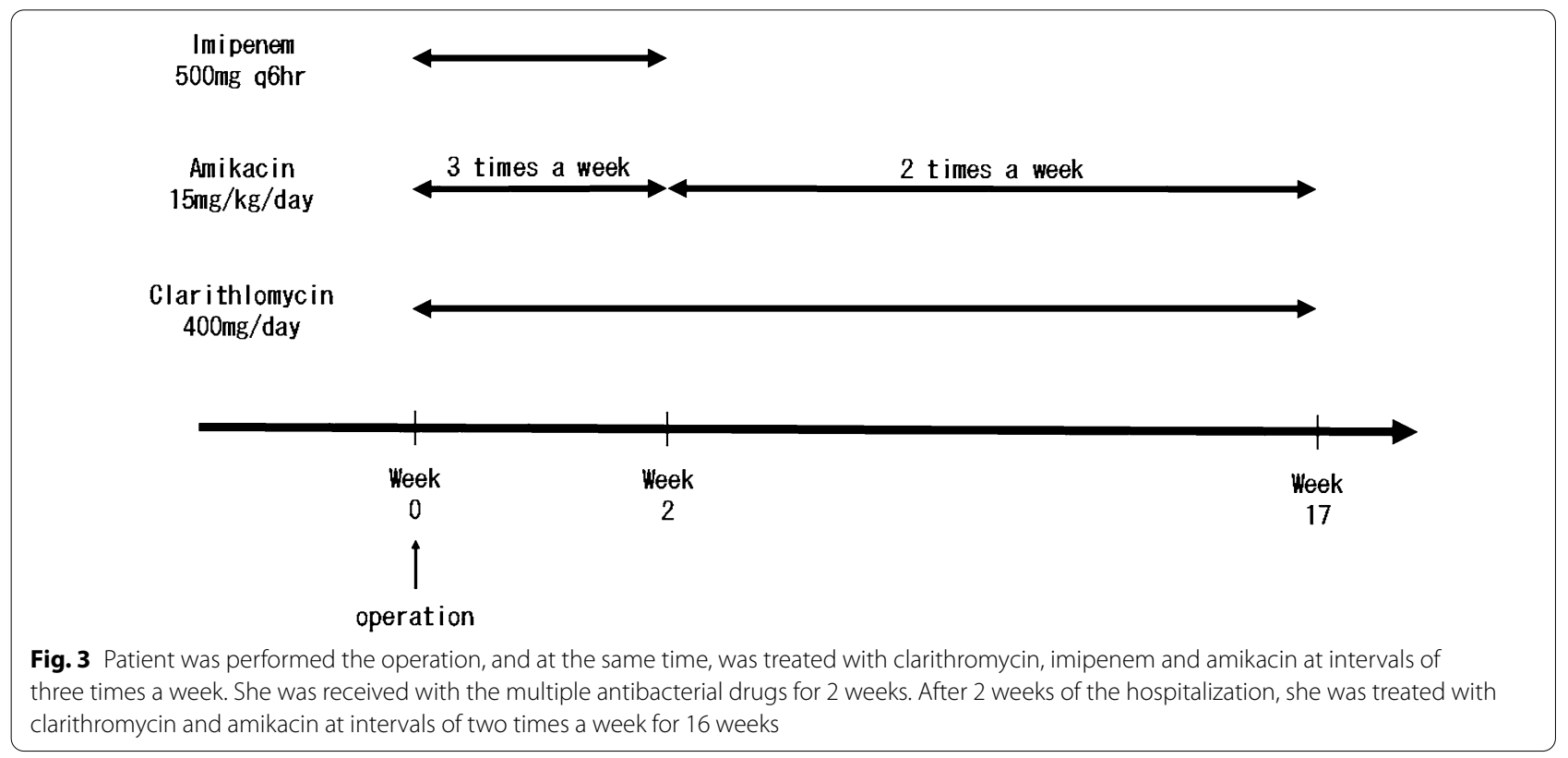


not immunodeficient, was infected by $M$. massiliense, because she had been repeatedly scratched around the nasopharynx with a machine using tap water.

\section{Diagnosis}

As NTM infections are very rare in the head and neck area, NTM infections are not often expected as a cause of nasopharyngitis. There may be more cases of pharyngeal NTM infections involved with pharyngitis that is resistant to treatment. We recommend that tissue samples are collected actively for the assessment of pharyngeal NTM infections where possible. It is important to preserve the quality and quantity of the samples for diagnosis [24]. Specifically, it is necessary to collect a sufficiently large specimen volume and to avoid exposure to bacteria from the surrounding environment, especially tap water, during collection. It is also a good idea to protect the specimen submission with sterile gauze soaked in reproduction to prevent it from drying out.

In this case, $M$. massiliense could not be identified by swab at first, and the biopsy sample could identify $M$. abscessus complex by culture examination. Although it may be difficult to obtain specimens from other parts of the body, such as the lungs, it is not difficult to obtain specimens from the head and neck region. Therefore, early biopsy is recommended for the diagnosis.

Because of differences in antimicrobial susceptibility in NTM infections, determining treatment policy is problematic, and species-level identification of the NTM is becoming increasingly important on a clinical level [24]. The PRA (Polymerase Chain Reaction-restriction enzyme pattern analysis) method identifies many NTM species that are not identifiable by phenotypic or chemotaxonomic techniques alone [1].

\section{Treatment}

M. abscessus complex isolates are uniformly resistant to standard anti-tuberculous agents [25-27] According to the American Thoracic Society/Infectious Diseases Society of America (ATS/IDSA), there is no reliable antibiotic regimen to produce a cure for the $M$. abscessus complex at present [1]. However, periodic administration of multidrug therapy, including a macrolide and one or more parenteral agents or a combination of parenteral agents over several months, may help control symptoms and the progression of $M$. abscessus complex disease [1]. In addition, it is reported that the only predictably curative therapy for focal $M$. abscessus complex disease is surgical resection combined with multidrug chemotherapy [1]. The treatment is limited to the current treatment policy for lung infections due to the problem with a lack of accumulated evidence.
The ATS/IDSA recommends the following chemotherapy for serious skin, soft tissue, and bone infections caused by the M. abscessus complex. Clarithromycin or azithromycin should be combined with parenteral medications (amikacin, cefoxitin, or imipenem) [26, 28]. The macrolides are the only oral agents found to be reliably active in vitro against the M. abscessus complex [26, 28].

The most active of the parenteral agents is amikacin [29]. Amikacin combined with high-dose cefoxitin is recommended for the initial therapy (minimum, 2 weeks) $[25,26,30]$. Therefore, hospitalization is recommended for a minimum of 2 weeks. However, limited cefoxitin availability may necessitate the choice of an alternative agent, such as imipenem [25, 26, 30]. For serious disease, a minimum of 16 weeks of therapy is necessary to increase the likelihood of a cure [1].

The clinical identification of $M$. massilience is significant due to the difference in therapeutic response between M. massiliense and other species [20]. A relatively large number of reports have described that even if treatment with the same treatment regimen is performed, the improvement rate in clinical findings, including imaging and physical findings, and symptoms are be better in M. massiliense than in other species [31-33]. The reason for the difference in response is the erm gene, which is a resistance-inducing gene for macrolide [34]. Thus, it may take less time to cure M. massiliense infection than other species [32].

In this case, treatment was performed according to the ATS/IDSA. Therefore, we did not use tigecycline. As the patient was exhausted by a few months of outpatient treatment, we had a discussion about the treatment policy with a doctor specializing in infectious diseases, including whether or not to switch to oral medicine. However, we decided to continue conventional treatment as far as possible. Eventually, the patient could not tolerate the outpatient treatment, and treatment ended after 16 weeks. It is possible to provide treatment that emphasizes the patient's quality of life if a shorter treatment period or an oral treatment regimen that is easy to continue is available that affords a better improvement rate in $M$. massiliense infections.

We reported the first case of pharyngeal $M$. massiliense infection. There may be more cases of pharyngeal NTM infections among cases of pharyngitis that are resistant to therapy. This patient underwent surgery and the multiple rounds of chemotherapy with oral and intravenous agents for 16 weeks, and no recurrence has been observed for the past 56 weeks. There is currently no definite treatment policy, and only empirical treatment is used; therefore, the further accumulation of data is expected in the future. 


\section{Abbreviations}

ATS/IDSA: American Thoracic Society/Infectious Diseases Society of America; M. abscessus: Mycobacterium abscessus; M. massiliense: Mycobacterium abscessus subspecies massiliense; NTM: Non-tubeculous mycobacteria; PCR: Polymerase chain reaction; RGM: Rapidly growing mycobacteria.

\section{Acknowledgements}

Not applicable.

\section{Authors' contributions}

$\mathrm{YO}, \mathrm{HH}, \mathrm{MK}, \mathrm{Yl}$ and $\mathrm{HI}$ examined and treated the patient. $\mathrm{YO}$ and $\mathrm{HH}$ wrote the manuscript and made all the figures. JT and $\mathrm{RH}$ advised on treatment protocol. NO supervised the manuscript. All authors read and approved the final manuscript.

\section{Funding}

H.H was supported by Grant-in-Aid for Scientific Research from the Ministry of Education, Science and Culture. The preparation of this article, English proofreading and publication fee were supported by JSPS KAKENHI Grant Number JP 18K09351.

\section{Availability of data and materials}

Not applicable.

\section{Declarations}

\section{Ethics approval and consent to participate}

Our institution's Research Ethics Board does not require a review or approval for case reports.

\section{Consent for publication}

The patient consented to the publication of this case report and written informed consent was obtained from the patient.

\section{Competing interests}

The authors declare that they have no competing interests.

\section{Author details}

${ }^{1}$ Department of Otolaryngology, Yokohama City University Medical Center, Urafune-cho 4-57, Minami-ku, Yokohama 232-0024, Japan. ${ }^{2}$ Department of Infectious Disease, Yokohama City University Medical Center, Urafune-cho 4-57, Minami-ku, Yokohama 232-0024, Japan. ${ }^{3}$ Department of Otolaryngology, Yokohama City University Graduate School of Medicine, 3-9 Fukuura, Kanazawa-ku, Yokohama 236-0004, Japan.

\section{Received: 11 March 2021 Accepted: 3 September 2021}

Published online: 18 September 2021

\section{References}

1. Griffith DE, et al. An official ATS/IDSA statement: diagnosis, treatment, and prevention of nontuberculous mycobacterial diseases. Am J Respir Crit Care Med. 2007;175(4):367-416.

2. Strnad L, Winthrop KL. Treatment of Mycobacterium abscessus complex. Semin Respir Crit Care Med. 2018;39(3):362-76.

3. Zelazny AM, et al. Cohort study of molecular identification and typing of Mycobacterium abscessus, Mycobacterium massiliense, and Mycobacterium bolletii. J Clin Microbiol. 2009:47(7):1985-95.

4. Atsuyuki K. Treatment of relatively rare species nontuberculous pulmonary mycobacteriosis. Kekkaku. 2011;86(12):923-32.

5. Lee MR, Sheng WH, Hung CC, Yu CJ, Lee LN, Hsueh PR. Mycobacterium abscessus complex infections in humans. Emerg Infect Dis. 2015;21(9):1638-46.

6. Myojin S, et al. Chronic otitis media caused by Mycobacterium abscessus spp. massiliense treated with tigecycline in a 10-year-old child. Int J Infect Dis. 2018;74:10-2.
7. Hotta O, Inoue N, Tanaka A, leiri N. Possible mechanisms underlying epipharyngeal abrasive therapy (EAT) with $\mathrm{ZnCl} 2$ solution for the treatment of autoimmune diseases and functional somatic syndrome. J Antivir Antiretrovir. 2017. https://doi.org/10.4172/1948-5964.1000168.

8. Osamu H, Chiyoko N. Various pathological conditions suggesting association of chronic epipharyngitis and the putative mechanism of epipharyngeal abrasive therapy. Jpn Soc Stomato Pharyngol. 2018;31(1):69-75.

9. Kazue N, et al. Discrimination of Mycobacterium abscessus subsp. massiliense from Mycobacterium abscessus subsp. abscessus in clinical isolates by multiplex PCR. J Clin Microbiol . 2014;52(1):251-9.

10. Mitsunori Y, Sotaro S, Jung CH, Hanako F, Masato S. A novel DNA chromatography method to discriminate Mycobacterium abscessus subspecies and macrolide susceptibility. EBioMedicine. 2021;64: 103187.

11. Wallace RJ Jr, Swenson JM, Silcox VA, Good RC, Tschen JA, Stone MS. Spectrum of disease due to rapidly growing mycobacteria. Rev Infect Dis. 1983. https://doi.org/10.1093/clinids/5.4.657.

12. Chandra NS, et al. Cluster of Mycobacterium chelonae keratitis cases following laser in-situ keratomileusis. Am J Ophthalmol. 2001;132(6):819-30.

13. Clegg HW, Foster MT, Sanders WE, Baine WB. Infection due to organisms of the Mycobacterium fortuitum complex after augmentation mammaplasty: clinical and epidemiologic features. J Infect Dis. 1983;147(3):427-33.

14. Wallace RJ, et al. Diversity and sources of rapidly growing mycobacteria associated with infections following cardiac surgery. J Infect Dis. 1989;159(4):708-16.

15. Wallace RJ, Zhang Y, Brown BA, Fraser V, Mazurek GH, Maloney S. DNA large restriction fragment patterns of sporadic and epidemic nosocomial strains of Mycobacterium chelonae and Mycobacterium abscessus. J Clin Microbiol. 1993;31(10):2697-701.

16. Lowry PW, et al. Mycobacterium chelonae causing otitis media in an earnose-and-throat practice. N Engl J Med. 1988;319(15):978-82.

17. Hector JSR, Pang Y, Mazurek GH, Zhang Y, Brown BA, Wallace RJ. Large restriction fragment patterns of genomic Mycobacterium fortuitum DNA as strain-specific markers and their use in epidemiologic investigation of four nosocomial outbreaks. J Clin Microbiol. 1992;30(5):1250-5.

18. Bolan G, et al. Infections with Mycobacterium chelonei in patients receiving dialysis and using processed hemodialyzers. J Infect Dis. 1985;152(5):1013-9.

19. Wallace RJ, Brown BA, Griffith DE. Nosocomial outbreaks/pseudooutbreaks caused by nontuberculous mycobacteria. Annu Rev Microbiol. 1998:52:453-90.

20. Daisuke J, et al. A case of infection with Mycobacterium massiliense in a patient who had long-term untreated Mycobacterium avium complex pulmonary disease. Kekkaku. 2017;92(6):463-7.

21. Falkinham JO. Nontuberculous mycobacteria in the environment. Clin Chest Med. 2002;23(3):529-51.

22. Von Reyn CF, et al. Isolation of Mycobacterium avium complex from water in the United States, Finland, Zaire, and Kenya. J Clin Microbiol. 1993;31(12):3227-30

23. Griffith DE, Girard WM, Wallace RJ. Clinical features of pulmonary disease caused by rapidly growing mycobacteria. An analysis of 154 patients. Am Rev Respir Dis. 1993;147(5):1271-8.

24. Wayne PA et al. Susceptibility testing of mycobacteria, nocardiae, and other aerobic actinomycetes Approved Standard. NCCLS. 2003;M24-A.

25. Brown BA, Wallace RJ, Onyi G, Rosas VDE, Wallace RJ. Activities of four macrolides, including clarithromycin, against Mycobacterium fortuitum, Mycobacterium chelonae, and M. chelonae-like organisms. Antimicrob Agents Chemother. 1992;36(1):180-4.

26. Swenson JM, Wallace RJ, Silcox VA, Thornsberry C. Antimicrobial susceptibility of five subgroups of Mycobacterium fortuitum and Mycobacterium chelonae. Antimicrob Agents Chemother. 2000;28(6):807-11.

27. Mary S, Christensen LA, Wallace RJ, Swenson JM, Thornsberry C. Agar disk elution method for susceptibility testing of Mycobacterium marinum and Mycobacterium fortuitum complex to sulfonamides and antibiotics. Antimicrob Agents Chemother. 1983;24(4):486-93.

28. Wallace RJ, Brown BA, Onyi G. Susceptibilities of Mycobacterium fortuitum biovar. fortuitum and the two subgroups of Mycobacterium chelonae to imipenem, cefmetazole, cefoxitin, and amoxicillin-clavulanic acid. Antimicrob Agents Chemother. 1991;35(4):773-5. 
29. Peloquin CA, et al. Aminoglycoside toxicity: daily versus thrice-weekly dosing for treatment of mycobacterial diseases. Clin Infect Dis. 2004 https://doi.org/10.1086/420742.

30. Wallace RJ, Brown BA, Onyi GO. Skin, soft tissue, and bone infections due to Mycobacterium chelonae chelonae: importance of prior corticosteroid therapy, frequency of disseminated infections, and resistance to oral antimicrobials other than clarithromycin. J Infect Dis. 1992;166(2):405-12.

31. Harada T, et al. Clinical and microbiological differences between Mycobacterium abscessus and Mycobacterium massiliense lung diseases. J Clin Microbiol. 2012;50(11):3556-61.

32. Lyu J, et al. A shorter treatment duration may be sufficient for patients with Mycobacterium massiliense lung disease than with Mycobacterium abscessus lung disease. Respir Med. 2014;108(11):1706-12.
33. Park J, Cho J, Lee CH, Han SK, Yim JJ. Progression and treatment outcomes of lung disease caused by Mycobacterium abscessus and Mycobacterium massiliense. Clin Infect Dis. 2017;64(3):301-8.

34. Koh W, et al. Clinical significance of differentiation of Mycobacterium massiliense from Mycobacterium abscessus. Am J Respir Crit Care Med. 2011;183(3):405-10

\section{Publisher's Note}

Springer Nature remains neutral with regard to jurisdictional claims in published maps and institutional affiliations.
Ready to submit your research? Choose BMC and benefit from:

- fast, convenient online submission

- thorough peer review by experienced researchers in your field

- rapid publication on acceptance

- support for research data, including large and complex data types

- gold Open Access which fosters wider collaboration and increased citations

- maximum visibility for your research: over 100M website views per year

At BMC, research is always in progress.

Learn more biomedcentral.com/submissions 